\title{
INNER MODEL OPERATORS AND THE CONTINUUM HYPOTHESIS
}

\author{
HOWARD BECKER ${ }^{1}$
}

\begin{abstract}
Assuming AD, every inner model operator satisfies the continuum hypothesis.
\end{abstract}

We work in $\mathrm{ZF}+\mathrm{DC}$, and usually assume the axiom of determinacy (AD). For an introduction to $\mathrm{AD}$, see [ 3 or 7]. In this paper, degree always means Turing degree. A cone of degrees is a set of the form $\left\{\mathbf{d}: \mathbf{d}_{0} \leqslant_{T} \mathbf{d}\right\}$. AD implies that the filter generated by the cones is a (countably complete) ultrafilter [7, 7D.15]. Almost everywhere (a.e.) will always refer to this measure. A real is a subset of $\omega$.

Definition. An inner model operator (or IMO) is a function $\mathscr{M}: x \mapsto\left(M_{x}, \leqslant_{x}\right)$ with domain $P(\omega)$ such that, for all reals $x, M_{x}$ is a transitive (set) model of ZFC, $\leqslant_{x}$ is a well-ordering of $P(\omega) \cap M_{x}$, and the following three properties hold:

(1) For any $x, y \subset \omega$, if $x \equiv_{T} y$ then $M_{x}=M_{y}$.

(2) For any $x \subset \omega, x \in M_{x}$.

(3) For any $x \subset \omega, f^{x} \in M_{x}$, where $f^{x}$ is the function with domain $\{y \subset \omega$ : $y \equiv_{T} x$ and such that $f^{x}(y)$ is $\leqslant_{y}$.

This concept was introduced by Steel [9]. The above definition is a variant of his. This concept is meant to be considered under the hypothesis of $\mathrm{AD}$, or some weak form of that axiom. Usually one identifies IMO's which agree almost everywhere. The canonical example of an IMO is the map $x \mapsto L(x)$. Any construction which relativizes to a real in a degree-invariant way gives an IMO. We give some other examples below. The definition is intended to be a formalization of the notion of a "natural" model; assuming $\mathrm{AD}$, it excludes many "unnatural" models such as forcing models.

Examples of inner model operators. 1. $x \mapsto L(x)$. This is an abuse of the language; what we really mean is $x \mapsto\left(L_{\kappa_{1}^{\prime}}(x), \leqslant_{L(x)}\right)$, where $\leqslant_{L(x)}$ is the canonical constructibility ordering. We will similarly abuse the language in all the following examples. All these examples have canonical well-orderings. In this paper we will only be concerned with reals and sets of reals in the model, so we might as well cut the universe off at a strongly inaccessible cardinal. (AD implies that $\boldsymbol{\aleph}_{1}$ will be strongly inaccessible in any transitive model of ZFC.)

2. $x \mapsto$ the minimal model of ZFC containing $x$.

3. $x \mapsto L\left(x^{\#}\right)$.

Received by the editors October 17, 1984.

1980 Mathematics Subject Classification. Primary 03E60; Secondary 03D80, 03E15, 03E45, 04A30.

${ }^{1}$ Research partially supported by NSF Grant MCS82-11328. 
4. More generally, for any jump operator $J, x \mapsto L(J(x))$ is an IMO. A jump

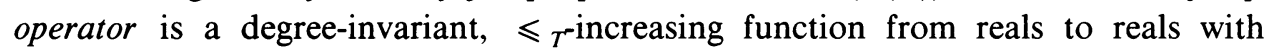
enough uniformity to make (3) in the definition of IMO hold. (We give a more precise definition later.)

5. $x \mapsto L[\mu, x]$, where $\mu$ is a fixed normal measure on some cardinal.

6. There are generalizations of the $L[\mu]$ construction to stronger large cardinal properties, for example Mitchell's models for coherent sequences of measurables and for hypermeasurables $[5,6]$. These give rise to IMO's.

7. $x \mapsto K(x)$, where $K(x)$ is the core model [2] relativized to $x$.

8. Assuming projective determinacy, there exist analogs of $L$ for the higher levels of the analytical hierarchy. For example, $P(\omega) \cap L$ is the largest countable $\Sigma_{2}^{1}$ subset of $P(\omega)$; analogously, for any $n$, there is a model $L^{2 n}$ such that $P(\omega) \cap L^{2 n}$ is the largest countable $\Sigma_{2 n}^{1}$ set. There are other classes of analogs, too, since generalizing different properties of $L$ gives different models. The following is a list of the analogs of $L$ which have appeared in the literature:

$M^{2}, M^{3}, M^{4}, M^{5}, \ldots \quad[1]$.

$L^{2}, L^{4}, L^{6}, L^{8}, \ldots \quad[1]$.

$H_{1}, H_{3}, H_{5}, H_{7}, \ldots \quad[7,8 \mathrm{G}]$.

$L\left(Q_{1}\right), L\left(Q_{3}\right), L\left(Q_{5}\right), L\left(Q_{7}\right), \ldots \quad[4]$.

$M^{2}=L^{2}=H_{1}=L\left(Q_{1}\right)=L$. These models all have canonical well-orderings and all relativize. Hence each of these models corresponds to an IMO.

9. There are also analogs of $L$, similar to those of Example 8, for pointclasses beyond the projective hierarchy, such as the inductive sets or $\left(\Sigma_{1}^{2}\right)^{L[\mathbf{R}]}$. These also give IMO's.

10. $x \mapsto \operatorname{HOD}(x)$.

Next we consider an example of something that is not an IMO. For the moment, work in $\mathrm{ZF}+\mathrm{DC}+(\forall x \subset \omega)$ ( $x^{\#}$ exists). Then for any $x \subset \omega$ there is a $y \subset \omega$ such that $y$ is Cohen-generic over $L(x)$. But is there an IMO $\mathscr{M}$ such that $M_{x}=L(x, y)$ where $y$ is Cohen-generic over $L(x)$ ? Assuming the axiom of choice the answer is obviously yes - for each degree d, just choose a $y$ and a well-ordering of $L(\mathbf{d}, y)$. (Without the axiom of choice, one can prove the existence of a function taking each $x$ to a $y$ which is generic over $L(x)$, for example, by taking the least $y$ with respect to the canonical well-ordering of $L(x \#)$. However, this function is not degree-invariant.) Without choice, it is conceivable that the answer is no.

Assuming AD, no such IMO exists. The following theorem of Steel [9] rules out this and many other forcing models: For any IMO $\mathscr{M}$, for a.e. $x$, either $(P(\omega) \cap$ $\left.M_{x}\right) \subset L(x)$ or $x^{\#} \in M_{x}$.

Definition. $\mathscr{M} \vDash \psi$ means that, for a.e. $x, M_{x} \vDash \psi$.

Note that under $\mathrm{AD}$, for any $\mathscr{M}$ and $\psi$, either $\mathscr{M} \vDash \psi$ or $\mathscr{M} \vDash \neg \psi$.

Theorem. Assume AD. Let $\mathscr{M}$ be an IMO. Then $\mathscr{M} \vDash C H$.

Remarks and questions. 1. Woodin has shown (assuming ZF $+\mathrm{DC}+(\forall x \subset \omega)$ ( $x^{\#}$ exists)) that there is an IMO $\mathscr{N}$ such that $\mathscr{N} \vDash 2^{\aleph_{2}}>\boldsymbol{N}_{3}$. This question is open for $2^{\aleph_{1}}=\aleph_{2}$ and also open for $\diamond$. Woodin's models $N_{x}$ are obtained by forcing 
over $L(x)$; the forcing used has the property that the generic object can be built in a degree-invariant way.

2. Assuming $\mathrm{AD}_{\mathrm{R}}$, there exist degree-invariant maps $x \mapsto M_{x}$ where $M_{x} \vDash(\mathrm{ZF}+$ $\neg \mathrm{AC})$, and in fact $M_{x} \vDash(\mathrm{ZF}+\mathrm{DC}+\mathrm{AD})$. This is proved in Solovay [8].

3. For examples (1)-(9) it was already known that $\mathrm{CH}$ holds in these models. Example (10) gives a new result. It was known that $\operatorname{HOD}^{L[R]} \vDash \mathrm{CH}$, and similarly relativized to $x$, but it was not known for other HOD's, e.g. the HOD of the world of $\operatorname{AD}_{\mathbf{R}}$ (which is not equal to $\mathrm{HOD}^{L[R]}$ ). The fact that $\operatorname{HOD}(x) \vDash \mathrm{CH}$, for a.e. $x$; leads to an obvious question: What about $x=0$ ? Assuming $\mathrm{ZF}+\mathrm{DC}+\mathrm{AD}_{\mathbf{R}}+$ $V=L[P(\mathbf{R})]$, does $\mathrm{HOD} \vDash \mathrm{CH}$ ?

4. Under any reasonable notion of definability, definable determinacy implies that definable IMO's satisfy $\mathrm{CH}$. For example, projective determinacy implies that, for any projective IMO $\mathscr{M}, \mathscr{M} \vDash \mathrm{CH}$. $\mathscr{M}$ is projective if the following two relations on reals are projective:

$$
\left\{(x, y): y \in M_{x}\right\}, \quad\left\{(x, y, z): y \in M_{x} \& z \in M_{x} \& y \leqslant_{x} z\right\} .
$$

Note that examples (1)-(3) and (5)-(8) are projective IMO's.

The rest of this paper consists of a proof of the theorem. We first need a sequence of lemmas. We work in ZF + DC, and state additional axioms in the hypotheses of the lemmas.

Lemma 1. Assume the axiom of choice. Let $C \subset P(\omega)$. Suppose that for any $x$, $y \in C$, either $x \leqslant_{T} y$ or $y \leqslant_{T} x$. Then $\operatorname{card}(C) \leqslant \aleph_{1}$.

Definition. A jump operator is a pair $(\mathbf{d}, J)$ where $\mathbf{d}$ is a degree and $J$ is a function from $\left\{x \subset \omega: \mathbf{d} \leqslant_{T} x\right\}$ into $P(\omega)$ such that:

(1) $J$ is uniformly degree-invariant, i.e. there is a function $f: \omega \rightarrow \omega$ such that, for all $e \in \omega$, for all $x, y \geqslant_{T}$ d, if $x \equiv_{T} y$ via $e$ then $J(x) \equiv_{T} J(y)$ via $f(e)$,

(2) $J$ is $\leqslant_{T}$-increasing, i.e., for all $x \geqslant_{T} \mathbf{d}, x \leqslant_{T} J(x)$.

Lemma 2 (Steel [9]). Assume AD. If $J_{1}$ and $J_{2}$ are jump operators, then for a.e. $x$, either $J_{1}(x) \leqslant{ }_{T} J_{2}(x)$ or $J_{2}(x) \leqslant{ }_{T} J_{1}(x)$.

Lemma 3. Let $\mathscr{M}$ be an IMO. There are functions $x \mapsto C_{x}, x \mapsto \xi_{x}$, and $x \mapsto \phi_{x}$ with the following properties:

(1) For all $x \subset \omega$,

(a) $C_{x} \subset\left(P(\omega) \cap M_{x}\right), \xi_{x}$ is an ordinal, and $\phi_{x}$ is a one-to-one function from $C_{x}$ onto $\xi_{x}$,

(b) $C_{x}, \xi_{x}$, and $\phi_{x}$ are in $M_{x}$,

(c) $M_{x} \vDash \operatorname{card}\left(C_{x}\right)=2^{\aleph_{0}}$,

(d) for all $z \in C_{x}, x \leqslant_{T} z$.

(2) For all $x, y \subset \omega$ such that $x \equiv_{T} y$,

(a) $\xi_{x}=\xi_{y}$

(b) for any $v$ and $w$, if $v \in C_{x}, w \in C_{y}$, and $\phi_{x}(v)=\phi_{y}(w)$ then $v \equiv{ }_{T} w$. Moreover, this is uniform in the following sense. There is a function $g: \omega \rightarrow \omega$ such 
that for any $v$ and $w$ and any $e \in \omega$, if $v \in C_{x}, w \in C_{y}, \phi_{x}(v)=\phi_{y}(w)$ and $x \equiv_{T} y$ via $e$, then $v \equiv_{T} w$ via $g(e)$.

Proof. Let $\mathscr{M}: x \mapsto\left(M_{x}, \leqslant_{x}\right)$ be an IMO. Let

$$
\xi_{x}=\sup \left\{\text { order type of } \leqslant_{y}: y \equiv_{T} x\right\} .
$$

For $\eta<\xi_{x}$, let $v_{x}^{\eta}$ be the real which is the join of $x^{\prime \prime}$ and

$$
\begin{aligned}
& \left\{(e, i) \in \omega^{2}:(\exists y, w \subset P(\omega))\left(y \equiv_{r} x \text { via } e \& w \in M_{x}\right.\right. \\
& \left.\left.\quad \& \text { the rank of } w \text { in } \leqslant_{y} \text { is } \eta \& i \in w\right)\right\} .
\end{aligned}
$$

Then let $C_{x}=\left\{v_{x}^{\eta}: \eta<\xi_{x}\right\}$ and let $\phi_{x}\left(v_{x}^{\eta}\right)=\eta$. This works. It follows from (2) and (3) in the definition of IMO that everything is in the model $M_{x}$. The reason (1c) holds is that every real in $M_{x}$ is recursive in an element of $C_{x}$.

Proof of Theorem. Fix an IMO $\mathscr{M}$ and fix maps $x \mapsto C_{x}, x \mapsto \xi_{x}$, and $x \mapsto \phi_{x}$ satisfying Lemma 3 for $\mathscr{M}$. By applying Lemma 1 inside the model $M_{x}$, we see that to show that $M_{x} \vDash \mathrm{CH}$, it will suffice to show that

$$
\left.\left(\forall z_{1}, z_{2} \in C_{x}\right) \text { (either } z_{1} \leqslant{ }_{T} z_{2} \text { or } z_{2} \leqslant{ }_{T} z_{1}\right) \text {. }
$$

Note that by part (2) of Lemma 3, $(*)$ depends only on the Turing degree of $x$. Suppose that the theorem is false. Then $(*)$ is false on a cone, say the cone above $\mathbf{d}_{0}$. For every $x \geqslant_{T} \mathbf{d}_{0}$, let $z_{1}(x)$ and $z_{2}(x)$ be the $\phi_{x}$-least counterexample to (*). By

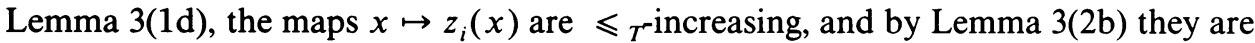
uniformly degree-invariant, that is, they are jump operators. For $x \geqslant_{T} \mathbf{d}_{0}, z_{1}(x) \star_{T}$ $z_{2}(x)$ and $z_{2}(x) \nless_{T} z_{1}(x)$. This contradicts Lemma 2.

\section{REFERENCES}

1. H. Becker, Partially playful universes, Cabal Seminar 76-77 (A.S. Kechris and Y. N. Moschovakis, eds.), Lecture Notes in Math., vol. 689, Springer-Verlag, 1978, pp. 55-90.

2. A. Dodd and R. Jensen, The core model, Ann. of Math. Logic 20 (1981), 43-75

3. T. J. Jech, Set theory, Academic Press, New York, 1978.

4. A. S. Kechris, D. A. Martin and R. M. Solovay, Introduction to Q-theory, Cabal Seminar 79-81 (A. S. Kechris, D. A. Martin and Y. N. Moschovakis, eds.), Lecture Notes in Math., vol. 1019, Springer-Verlag, 1983, pp. 199-281.

5. W. Mitchell, Sets constructible from sequences of ultrafilters, J. Symbolic Logic 39 (1974), 57-66.

6. Hypermeasurable cardinals (M. Boffa, D. Van Dalen and K. McAloon, eds.), Logic Colloquium '78, North-Holland, Amsterdam, 1979, pp. 303-316.

7. Y. N. Moschovakis, Descriptive set theory, North-Holland, Amsterdam, 1980.

8. R. M. Solovay, The independence of DC from AD, Cabal Seminar 76-77 (A. S. Kechris and Y. N. Moschovakis, eds.), Lecture Notes in Math., vol. 689, Springer-Verlag, 1978, pp. 171-184.

9. J. R. Steel, A classification of jump operators, J. Symbolic Logic 47 (1982), 347-358.

Department of Mathematics, University of South Carolina, Columbia, South Carolina 29208 (Current address)

Department of Mathematics, California Institute of Technology, Pasadena, California 91125 